\title{
MULTIPLE STABLE SOLUTIONS OF NONLINEAR BOUNDARY VALUE PROBLEMS ARISING IN CHEMICAL REACTOR THEORY*
}

\author{
DONALD S. COHEN $\dagger$
}

1. Introduction. Recent experimental and theoretical results show that an adiabatic tubular reactor, in which there is occurring a simple first order irreversible exothermic chemical reaction, can exhibit multiple stable steady states. The papers of R. Aris [1] and D. Luss [2] list most of the pertinent chemical engineering literature. Mathematically, the problem to be studied is the following nonlinear boundary value problem:

$$
\begin{aligned}
\beta u^{\prime \prime}-u^{\prime}+f(u) & =0, & 0 \leqq x \leqq 1, \\
u^{\prime}(0)-a u(0) & =0, & \\
u^{\prime}(1) & =0 . &
\end{aligned}
$$

The function $u$ represents the dimensionless temperature in the reactor, and $\beta$ and $a$ are known constants. The function $f(u)$, which essentially represents the rates of chemical production of the species (or equivalently, the rate of heat generation) in the reactor, is the Arrhenius reaction rate given by

$$
f(u)=b(c-u) \exp \frac{-k}{1+u} .
$$

The concentrations of the various chemical species involved in the reaction can be determined in a simple manner from a knowledge of $u$ and the stoichiometric coefficients of the species.

For simplicity we shall confine our analysis to the problem (1.1)-(1.4). However, for the most part our results also apply to the more general problem

$$
\begin{array}{lrl}
L u & =-F(x, u), & x \in D, \\
B u & =0, & x \in \partial D,
\end{array}
$$

where $B$ is a linear boundary operator, $L$ is a uniformly elliptic second order partial differential operator for which the strong maximum principle holds, and $F(x, u)$, for fixed $x$, "resembles" $f(u) . D$ and $\partial D$ denote respectively the domain and its boundary.

In $\S 2$ a formal singular perturbation procedure is applied for one physically interesting range of the parameters. Our procedure clearly reveals the mechanism by which one, two or three solutions of (1.1)-(1.4) can occur and also the mechanism which governs discontinuities or "jump phenomena" in the control of the chemical process.

In $\S 3$ we introduce iteration procedures, defined by linear equations, which yield sequences which converge monotonically to the maximal (largest) solution

* Received by the editors January 5, 1970.

$\uparrow$ Department of Applied Mathematics, California Institute of Technology, Pasadena, California 91109. This work was performed at the Technological University of Denmark and was supported in part by a grant from Statens naturvidenskabelige Forskningsraad. 
from above and to the minimal (smallest) solution from below. Also, conditions both for uniqueness and for the appearance of multiple solutions are given. Taking these together with the results of $\S 2$, we can give a simple necessary and sufficient condition for multiplicity in the reactor.

Finally, in $\S 4$ we examine the stability of the different solutions when considered as steady states of the transient (parabolic) problem for which (1.1) (1.4) describes the corresponding steady state. Sufficient conditions for stability are given, and for certain ranges of the parameters it is shown that a unique solution is always stable, and when there are three solutions, the upper and lower ones are stable. Thus the sequences of $\S 2$ converge to stable steady state solutions.

2. Multiple solutions. Throughout this section (and only in this section) we shall assume that $0<\beta \ll 1$. (In chemical reactor theory this is the case when the Peclet number is large.) We now construct asymptotic expansions of the solutions of (1.1)-(1.4) as $\beta \rightarrow 0$ by standard techniques [3]. In all cases $f(u)=O(1)$, and there is a boundary layer of thickness $O(\beta)$ near $x=1$. Away from this boundary layer the first term of the expansion (the outer expansion) is given by

$$
\begin{aligned}
-u^{\prime}+f(u) & =0, & 0 \leqq x<1, \\
u^{\prime}(0)-a u(0) & =0 . &
\end{aligned}
$$

Evaluating (2.1) at $x=0$, we find that (2.1) and (2.2) together imply that

$$
a u(0)=f(u(0)) .
$$

Clearly, the solutions of (2.3) provide the proper initial conditions for (2.1). Figure 1 illustrates the solutions of $(2.3)$; we have shown curves of $f(u)$ for five different sets of values of $b$ and $k$ with $c$ fixed.

It is clear from the figure that $u(0)$ can assume one, two or three values. We shall discuss the implications and the conditions on $a, b$ and $k$ which determine the number of intersections later. At present we continue with the construction of the asymptotic expansion. Denote the solutions of (2.3) by $u(0)=\alpha_{i}$, where $i$ can assume the values 1,2 and/or 3. Then, the first term in the outer expansion is given by

$$
\begin{aligned}
-v^{\prime}+f(0) & =0, \\
v(0) & =\alpha_{i} .
\end{aligned}
$$

In the boundary layer we introduce a new length $\tilde{x}=(1-x) / \beta$ and let $u(x) \equiv u(1-\beta \tilde{x}) \equiv w(\tilde{x})$. Then, the first term of the expansion (the inner expansion) near $x=1$ is given by

$$
\begin{aligned}
w^{\prime \prime}+w^{\prime} & =0, \\
w^{\prime}(0) & =0, \\
w(\infty) & =v(1) .
\end{aligned}
$$

The boundary condition (2.8) expresses the proper condition for matching the inner and outer expansions. The solution of $(2.6)-(2.8)$ is $w(\tilde{x}) \equiv v(1)$, and thus the solution $v(x)$ of $(2.4),(2.5)$ is a uniformly valid expansion on $0 \leqq x \leqq 1$ to $O(\beta)$. 


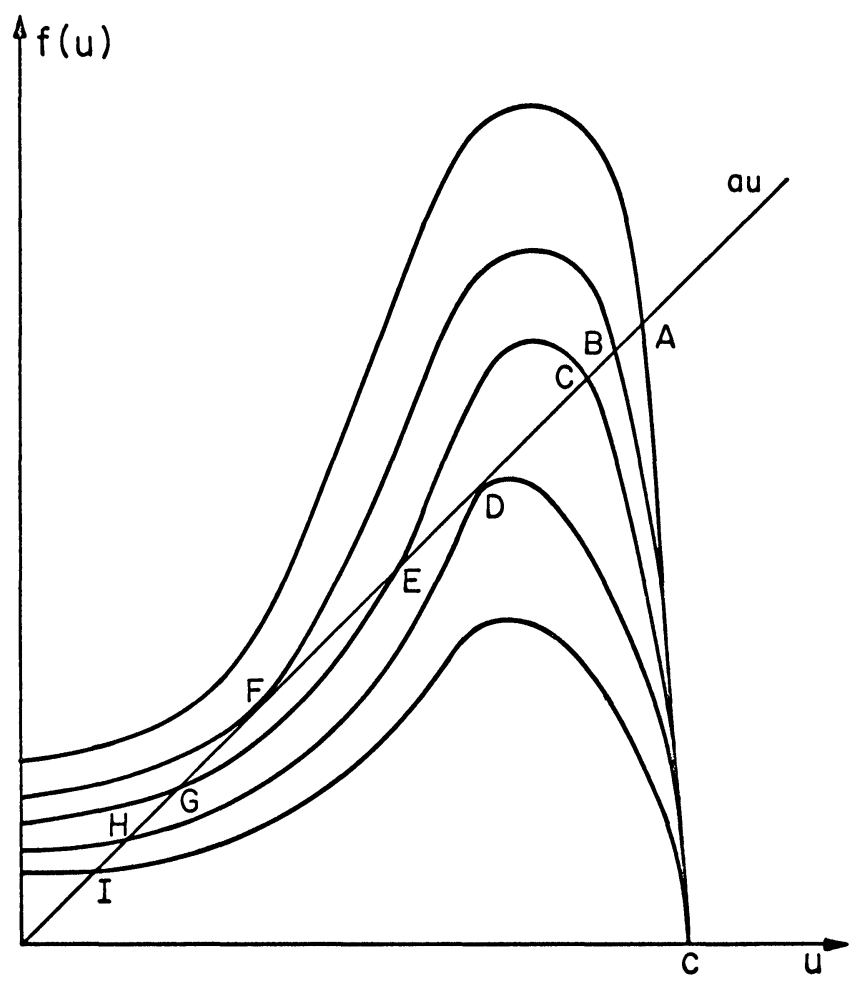

FIG. 1

We wish to make the following two points: (i) If, as sometimes occurs in practice, $a=O(1 / \beta)$ of $f^{\prime}(u)=O(1 / \beta)$ for some $u$, our procedure for constructing the first term of the asymptotic expansion is still valid. Of course, we would then expect differences in the higher terms. (ii) It is obvious from physical considerations and we show analytically in $\S 3$ that all solutions $u(x)$ of $(1.1)-(1.4)$ are such that $u^{\prime}(x) \geqq 0$ and $0 \leqq u(x) \leqq c$ for $0 \leqq x \leqq 1$. In order to see that the solution $v(x)$ of (2.4), (2.5) also possesses these properties, integrate (2.4) with respect to $x$ from 0 to 1 and use (2.5) to obtain

$$
\int_{\alpha_{i}}^{v(1)} \frac{d v}{f(v)}=1 .
$$

$v(1)$ is the quantity which must be found from (2.9) to be used in the matching condition (2.8). If $v(1)=\alpha_{i}$, the left-hand side of (2.9) is zero, and if $v(1)=c$, the left-hand side of (2.9) is infinite since the form (1.4) of $f(u)$ shows that the integral diverges logarithmically as $v(1) \rightarrow c$. Thus, since the integral is a continuous monotonic function of $v(1),(2.9)$ has exactly one solution $v(1)$ such that $\alpha_{i}<v(1)<c$, which then implies that $v^{\prime}(x) \geqq 0$ and $0 \leqq v(x) \leqq c$ on $0 \leqq x \leqq 1$.

Equation (2.3) and its geometrical interpretation in Fig. 1 allow us to obtain in the present problem the same sort of information which has been obtained [1] for simpler chemical reactors (such as the stirred tank). The curves in the figure move down as $k$ increases and move up as $b$ increases. Therefore, the number of intersections of the line $a u$ with the curve $f(u)$ can be changed by varying any or 
all of $a, b$ and $k$. Suppose, for example, that we fix $a$ and $k$ and vary $b$ starting on the top curve. Proceeding in order with the five curves illustrated in Fig. 1, we obtain respectively one, two, three, two and one steady states. (In practice the flow rate in the reactor can easily be changed independent of the other parameters, and $a$ is directly proportional to the flow rate while $b$ is inversely proportional to the flow rate. Thus, the multiplicity of solutions proceeds just as we have described when the flow rate is monotonically changed.)

It is also possible to obtain a "jump phenomenon" typical of many nonlinear response curves. Suppose we start our process at $A$ and increase the flow rate so that $a$ increases and $b$ decreases. Clearly, we proceed from $A$ to $B$ to $C$ to $D$. Then, the slightest increase in flow rate from that at $D$ gives an intersection near $H$ which clearly lies at a much lower temperature than $D$. (This violent jump to much lower temperatures corresponds to quenching the reaction by too great a flow rate.) A further increase in flow rate produces the change from $H$ to $I$. If we reverse the process by decreasing the flow rate, we proceed through the sequence $I, H, G, F$. Then, a further decrease in flow rate forces a jump to an intersection near $B$ (corresponding to sudden ignition).

3. Maximal and minimal solutions. In this section we shall introduce iteration schemes which converge to the maximal (largest) solution from above and to the minimal (smallest) solution from below. Therefore, among other things, we give rigorous existence proofs of maximal and minimal solutions of (1.1)-(1.4). Furthermore, conditions for uniqueness and for the appearance of multiple solutions are given. First, for brevity in later arguments we define the linear operator $L$ by $L u \equiv \beta u^{\prime \prime}-u^{\prime}$, and we refer to the boundary conditions (1.2) and (1.3) as $B u=0$. Thus, $B$ is a linear boundary operator, and the problem (1.1)-(1.4) can be written as

$$
L u=-f(u), \quad B u=0 .
$$

Basic for all our work is the following lemma.

Positivity Lemma. Let $\Omega$ be a nonnegative constant, and let $\phi(x)$ be twice continuously differentiable and satisfy

$$
L \phi-\Omega \phi \leqq 0, \quad B \phi=0 .
$$

Then, either $\phi(x) \equiv 0$ on $0 \leqq x \leqq 1$ or $\phi(x)>0$ for $0<x<1$.

This result is a consequence of the strong maximum principle for elliptic equations [4]; or, to prove it directly for the special operators $L-\Omega$ and $B$ of the present problem, construct the Green's function $G$, write (3.2) as an integral equation with $G$ as kernel, and note that $G$ is negative.

A further preliminary result is the following theorem.

THEOREM 3.1. Every solution $u(x)$ of (1.1)-(1.4) satisfies $0 \leqq u(x) \leqq c$ and $u^{\prime}(x) \geqq 0$ on $0 \leqq x \leqq 1$.

Proof. The bound $0 \leqq u(x) \leqq c$ is an immediate consequence of Theorem 2.1 of Cohen and Laetsch [5]. The positivity of $u^{\prime}(x)$ follows from writing $\beta u^{\prime \prime}-u^{\prime}$ as

$$
\beta e^{x / \beta} \frac{d}{d x}\left(e^{-x / \beta} u^{\prime}\right)
$$


integrating (1.1) with respect to $x$ from $x$ to 1 , and using (1.3) to obtain

$$
u^{\prime}(x)=\frac{1}{\beta} e^{x / \beta} \int_{x}^{1} e^{-x / \beta} f(u(x)) d x .
$$

Since $f(u) \geqq 0$ for $0 \leqq u \leqq c$, it follows that $u^{\prime}(x) \geqq 0$ on $0 \leqq x \leqq 1$.

The a priori bound $0 \leqq u(x) \leqq c$ can be used to replace $f(u)$ by one which coincides with $f(u)$ on $0 \leqq u \leqq c$, which is bounded and nonnegative for all $u$, and for which the extension is as smooth as we please. We now state that throughout the remainder of this section we are replacing $f(u)$ by one with these properties. Thus, we can find positive numbers $M$ and $\Omega$ such that for all $u$,

$$
\begin{gathered}
0 \leqq f(u) \leqq M, \\
\inf _{0 \leqq x \leqq 1} f^{\prime}(u)>-\Omega .
\end{gathered}
$$

Now, define sequences $\left\{u_{n}(x)\right\}$ and $\left\{v_{n}(x)\right\}$ by

$$
\begin{gathered}
L u_{0}=-M, \quad B u_{0}=0, \\
L u_{n}-\Omega u_{n}=-f\left(u_{n-1}\right)-\Omega u_{n-1}, \quad B u_{n}=0, \quad n=1,2,3, \cdots,
\end{gathered}
$$

and

$$
\begin{gathered}
v_{0}(x) \equiv 0, \\
L v_{n}-\Omega v_{n}=-f\left(v_{n-1}\right)-\Omega v_{n-1}, \quad B v_{n}=0, \quad n=1,2,3, \cdots .
\end{gathered}
$$

THEOREM 3.2. $u_{n}(x)>0$ and $v_{n+1}(x)>0$ for all $n \geqq 0$.

Proof. The proof is by induction. The Positivity Lemma immediately implies that $u_{0}(x)>0$. Assume $u_{v}(x)>0$ for all $v \leqq n-1$. Then,

$$
L u_{n}-\Omega u_{n}=-f\left(u_{n-1}\right)-\Omega u_{n-1}<0, \quad B u_{n}=0 .
$$

Hence, it follows from the Positivity Lemma that $u_{n}(x)>0$. Clearly, the same proof applies for the $v_{n}(x)$ also.

THEOREM 3.3. The sequence $\left\{u_{n}(x)\right\}$ is monotone nonincreasing, and the sequence $\left\{v_{n}(x)\right\}$ is monotone nondecreasing; that is,

$$
u_{n+1}(x) \leqq u_{n}(x) \text { and } v_{n+1}(x) \geqq v_{n}(x) \quad \text { for } n=0,1,2, \cdots .
$$

Furthermore, $v_{n}(x) \leqq u_{0}(x)$ for all $n \geqq 0$.

Proof. The proof is by induction. Equations (3.9) and (3.10) imply

$$
L\left(v_{1}-v_{0}\right)-\Omega\left(v_{1}-v_{0}\right)=-f(0)<0, \quad B\left(v_{1}-v_{0}\right)=0 .
$$

Hence, from the Positivity Lemma we conclude that $v_{1}(x)-v_{0}(x) \geqq 0$. Now, assume that $v_{v}(x)-v_{v-1}(x) \geqq 0$ for all $v \leqq n$. Then, (3.10) implies that

$$
\begin{aligned}
L\left(v_{n+1}-v_{n}\right)-\Omega\left(v_{n+1}-v_{n}\right) & =f\left(v_{n-1}\right)-f\left(v_{n}\right)-\Omega\left(v_{n}-v_{n-1}\right) \\
& =f^{\prime}(\zeta)\left(v_{n-1}-v_{n}\right)-\Omega\left(v_{n}-v_{n-1}\right) \\
& =-\left[f^{\prime}(\zeta)+\Omega\right]\left(v_{n}-v_{n-1}\right) \leqq 0,
\end{aligned}
$$

where $v_{n-1} \leqq \zeta \leqq v_{n}$ and we have obviously used the mean value theorem and then 
inequality (3.6). Since $B\left(v_{n+1}-v_{n}\right)=0$, the Positivity Lemma implies $v_{n+1}(x)$ $-v_{n}(x) \geqq 0$, and thus $\left\{v_{n}(x)\right\}$ is monotone nondecreasing. To show that the $\left\{u_{n}(x)\right\}$ are monotone nonincreasing, we note that (3.7) and (3.5) imply that $L\left(u_{0}-u_{1}\right)-\Omega\left(u_{0}-u_{1}\right)=-\left(M-f\left(u_{0}\right)\right) \leqq 0, B\left(u_{0}-u_{1}\right)=0$. Thus, $u_{0}(x)$ $-u_{1}(x) \geqq 0$, and clearly the rest follows by induction just as for the $v_{n}(x)$. follows :

To prove that the $v_{n}(x)$ are uniformly bounded above by $u_{0}(x)$ we proceed as

$$
L v_{1}-\Omega v_{1}=-f(0) \geqq-M=L u_{0} .
$$

Thus,

$$
L\left(u_{0}-v_{1}\right) \leqq 0, \quad B\left(u_{0}-v_{1}\right)=0,
$$

so that the Positivity Lemma implies $u_{0}(x)-v_{1}(x) \geqq 0$. Now, assume that $u_{0}(x)-v_{v}(x) \geqq 0$ for all $v \leqq n-1$. Then,

$$
L v_{n}-\Omega v_{n}=-f\left(v_{n-1}\right)-\Omega v_{n-1} \geqq-M-\Omega v_{n-1}=L u_{0}-\Omega v_{n-1} .
$$

Thus, (3.16) and the monotonicity of the $v_{n}(x)$ imply that

$$
L\left(u_{0}-v_{n}\right) \leqq \Omega\left(v_{n-1}-v_{n}\right) \leqq 0, \quad B\left(u_{0}-u_{n}\right)=0 .
$$

Hence, from the Positivity Lemma we conclude that $v_{n}(x) \leqq u_{0}(x)$.

The existence of maximal and minimal solutions is established by the next theorem.

THEOREM 3.4. The sequence $\left\{u_{n}(x)\right\}$ converges to the maximal solution $\tilde{u}(x)$ of (1.1)-(1.4) and the sequence $\left\{v_{n}(x)\right\}$ converges to the minimal solution $v(x)$ of $(1.1)-(1.4)$; that is, $\tilde{u}(x) \geqq u(x)$ and $v(x) \leqq u(x)$ for any solution $u(x)$.

Proof. We give the proof only for the sequence $\left\{u_{n}(x)\right\}$; with the obvious modifications the same proof applies to the $v_{n}(x)$.

Having demonstrated in Theorems 3.2 and 3.3 that the sequence $\left\{u_{n}(x)\right\}$ is monotone nonincreasing and bounded from below, we may immediately conclude that there is a limit function, say

$$
\lim _{n \rightarrow \infty}\left[u_{n}(x)\right]=\tilde{u}(x) .
$$

That $\tilde{u}(x)$ is a solution of (1.1)-(1.4) follows from the work of R. B. Simpson and D. S. Cohen [6] for more general problems of the form (1.5), (1.6). They used fixedpoint theorems and Schauder-type estimates from the theory of elliptic equations. For the special operators $L$ and $B$ of the present problem, however, we can also give the following simpler proof that $\tilde{u}(x)$ is a solution of $(1.1)-(1.4)$ : Write the iteration scheme (3.8) equivalently as

$$
u_{n}(x)=\int_{0}^{1} G(x, \xi)\left[-f\left(u_{n-1}(\xi)\right)-\Omega\left(u_{n-1}(\xi)-u_{n}(\xi)\right)\right] d \xi, \quad n=1,2,3, \cdots,
$$

where $G(x, \xi)$ is the Green's function for $L$ subject to the boundary conditions that $B u_{n}=0$. Since the $u_{n}(x)$ and $f\left(u_{n}(x)\right)$ are bounded, the integrand in (3.19) can clearly be bounded by an integrable function independent of $u_{n}$. Thus, the Lebesque bounded convergence theorem for Riemann integrals implies that the limit can be 
taken under the integral in (3.19) to conclude that

$$
\tilde{u}(x)=-\int_{0}^{1} G(x, \xi) f(\tilde{u}(\xi)) d \xi .
$$

It follows that $\tilde{u}(x)$ is a solution of (1.1)-(1.4).

We now show that $\tilde{u}(x)$ is maximal. Assume that $u(x)$ is any solution. Then, $u(x)$ satisfies $L u=-f(u), B u=0$. This fact and (3.7) imply

$$
L\left(u_{0}-u\right)=-(M-f(u)) \leqq 0, \quad B\left(u_{0}-u\right)=0 .
$$

The Positivity Lemma immediately implies that $u_{0}(x)-u(x) \geqq 0$. We now proceed by induction. Assume that $u_{v}(x)-u(x) \geqq 0$ for all $v \leqq n-1$. Then,

$$
\begin{aligned}
L\left(u_{n}-u\right) & =\Omega\left(u_{n}-u_{n-1}\right)-f\left(u_{n-1}\right)+f(u) \\
& =\Omega\left(u_{n}-u+u-u_{n-1}\right)-f^{\prime}(\eta)\left(u_{n-1}-u\right),
\end{aligned}
$$

where we have used the mean value theorem and $u \leqq \eta \leqq u_{n-1}$. Hence,

$$
\begin{aligned}
L\left(u_{n}-u\right)-\Omega\left(u_{n}-u\right) & =\Omega\left(u-u_{n-1}\right)-f^{\prime}(\eta)\left(u_{n-1}-u\right) \\
& =-\left[\Omega+f^{\prime}(\eta)\right]\left(u_{n-1}-u\right) \leqq 0,
\end{aligned}
$$

and $B\left(u_{n}-u\right)=0$. Therefore, from the Positivity Lemma we conclude that $u_{n}(x)-u(x) \geqq 0$; that is, $\left\{u_{n}(x)\right\}$ converges to the maximal solution.

The formal analysis of $\S 2$ indicates that multiple solutions may exist. In fact, for certain sets of constants $\beta, a, b, c, k, \mathrm{~L}$. R. Raymond and N. R. Amundson [10] and N. R. Amundson and D. Luss [12] have numerically computed three different solutions. Hence, $\tilde{u}(x)$ and $v(x)$ can represent different solutions. The general problem (1.5), (1.6) with various classes of nonlinearities $F(x, u)$ has been investigated recently [5]-[9] with particular regard to existence, uniqueness, multiplicity, and bifurcation phenomena. It has become clear from these studies that certain quantities (and their geometrical interpretations) play a crucial role in the answers to these questions. For the present problem (1.1)-(1.4) an immediate consequence of Theorem 4.2 of D. S. Cohen and T. W. Laetsch [5] is the following theorem.

THEOREM 3.5. If the constants $b, c$ and $k$ are such that

$$
\frac{d}{d u}\left[\frac{f(u)}{u}\right]<0
$$

for $0 \leqq u \leqq c$, then the solution of (1.1)-(1.4) is unique.

For the nonlinearity (1.4) the condition (3.24) has the following two interesting geometrical interpretations : (i) any line segment from the origin to the function lies below the graph of the function; and (ii) the tangent to the curve $y=f(u)$ on $0 \leqq u \leqq c$ intersects the axis of ordinates (i.e., the $u=0$ axis) in $y>0$. Our formal analysis of $\S 2$ indicates that it is precisely when these conditions are violated that uniqueness is lost and multiple solutions appear. Hence, for the present problem the condition (3.24) for uniqueness is a sharp dividing line. Thus, it appears to be necessary and sufficient that $(d / d u)[f(u) / u]$ change sign for multiple solutions to exist. We make this claim only for the function $f(u)$ given by (1.4); counterexamples can easily be constructed for other nonlinearities. 
4. Stability. We shall now give sufficient conditions for the stability of the different solutions. In particular, for various ranges of the parameters we show that a unique solution is always stable, and when there are three solutions, the upper and lower ones can both be stable.

It is shown in [11] that the solutions of $(1.1)-(1.4)$ are the steady state solutions of the parabolic problem

$$
\begin{aligned}
\beta U_{x x}-U_{x}+f(U) & =\beta U_{t}, \quad 0 \leqq x \leqq 1, \quad t \geqq 0, \\
U_{x}(0, t)-a U(0, t) & =0, \\
U_{x}(1, t) & =0, \\
U(x, 0) & =U_{0}(x) .
\end{aligned}
$$

We shall give a more precise definition later, but roughly, we say that a solution $u(x)$ of $(1.1)-(1.4)$ is stable if for all initial data of the form

$$
U_{0}(x)=u(x)+\varepsilon V(x),
$$

the solution of (4.1)-(4.4) decays exponentially in $t$ to $u(x)$ to first order in $\varepsilon$.

Assuming a solution of (4.1)-(4.4) of the form

$$
U(x, t)=u(x)+\varepsilon v(x) e^{-\sigma t}+O\left(\varepsilon^{2}\right),
$$

we find, to first order in $\varepsilon$, that $\sigma$ and $v(x)$ must satisfy

$$
\begin{aligned}
\beta v^{\prime \prime}-v^{\prime}+\left[\sigma \beta+f^{\prime}(u)\right] v & =0, \\
v^{\prime}(0)-a v(0) & =0, \\
v^{\prime}(1) & =0 .
\end{aligned}
$$

Clearly, nontrivial solutions $v \not \equiv 0$ exist if and only if $\sigma$ is an eigenvalue of (4.7)-(4.9) and $v=v(\sigma, x)$ is the corresponding eigenfunction. The eigenfunctions are complete, and thus, for some coefficients $h_{n}$,

$$
V(x)=\sum_{n} h_{n} v\left(\sigma_{n}, x\right)
$$

and the solution, to first order in $\varepsilon$, of (4.1) (4.4) is

$$
U(x, t)=u(x)+\varepsilon \sum_{n} h_{n} v\left(\sigma_{n}, x\right) e^{-\sigma_{n} t}+O\left(\varepsilon^{2}\right) .
$$

Thus, we are motivated to adopt the following definition.

Definition. A solution $u(x)$ of (1.1)-(1.4) is stable if the first eigenvalue $\sigma=\sigma_{1}$ of (4.7)-(4.9) is positive; it is unstable if $\sigma_{1}$ is negative; and it is neutrally stable if $\sigma_{1}=0$.

For our analysis it is convenient to let $v=y e^{x /(2 \beta)}$. Then, we find that

$$
\begin{array}{r}
y^{\prime \prime}+[\sigma-q(x)] y=0, \\
y^{\prime}(0)-\left(a-\frac{1}{2 \beta}\right) y(0)=0, \\
y^{\prime}(1)+\frac{1}{2 \beta} y(1)=0,
\end{array}
$$


where

$$
q(x)=\frac{1}{4 \beta^{2}}-\frac{1}{\beta} f^{\prime}(u(x)) .
$$

In the chemical reactor theory it is always the case that $a-1 /(2 \beta)>0$, and hence we shall maintain this condition here. Our problem now is to determine sufficient conditions for the first eigenvalue $\sigma_{1}$ of (4.12)-(4.15) to be positive.

It is well known that if $q(x)$ is positive everywhere in the interval $0 \leqq x \leqq 1$, then all eigenvalues of (4.12)-(4.15) are positive. Hence, an immediate consequence of this is the result that if the constants $b, c$ and $k$ are such that $f^{\prime}(u)=O(1)$ for $0 \leqq u \leqq c$, then for $\beta$ sufficiently small, all solutions of (1.1)-(1.4) are stable. In practice, it is generally the case that $\beta$ small and $f^{\prime}(u)=O(1)$ imply only one solution of (2.3) and hence a unique solution of (1.1)-(1.4). Thus, in this case the unique solution is stable.

We shall now show that $q(x)$ is positive for both the upper and lower solutions in a special case where three solutions exist for $\beta$ of moderate size. More precisely, Amundson and Luss [12] have computed the three solutions for the situation where $\beta=5, a=0.2$ and

$$
f(u)=2\left(10^{7}\right)(0.4-u) e^{-20 /(1+u)} .
$$

(The results are given in Example 1 and Fig. 7 of [12].) Three solutions $u_{3}(x)$ $>u_{2}(x)>u_{1}(x)$ are found such that $0.01<u_{1}(x)<0.03$ and $0.32<u_{3}(x)<0.37$. It is a simple computation to show that $f^{\prime}\left(u_{3}(x)\right)<0$ and $f^{\prime}\left(u_{1}(x)\right)<1 /(4 \beta)$ for $0 \leqq x \leqq 1$. Hence, $q(x)$ is positive for both $u_{1}(x)$ and $u_{3}(x)$, and therefore, both $u_{1}(x)$ and $u_{3}(x)$ are stable solutions.

A more difficult situation which commonly occurs (see [10] and [12] for experimental values of the parameters) is the case where $\beta$ is small and $f(u)=O(1)$ on $0 \leqq u \leqq c$ with $f^{\prime}(u)=O(1 / \beta)$ somewhere in $0 \leqq u \leqq c$. The function $q(x)$ is then found to assume negative values on some subinterval of $0 \leqq x \leqq 1$, and then both the magnitude of the negative values and the length of the interval on which they are assumed play critical roles in determining the sign of $\sigma_{1}$. We shall now study this case.

First, we give a formal analysis correct to terms of order $O(\beta)$ based on the singular perturbation procedure of $\S 2$. Note that $a-1 /(2 \beta)>0$ and $0<\beta \ll 1$ implies that $a$ is at least of order $O(1 / \beta)$, and hence the straight line in Fig. 1 is of such large slope that (2.3) has only one solution $\alpha=\alpha_{1}$, where $\alpha_{1}$ is close to zero since $a$ is large. This solution $u_{1}(x)$ and the corresponding $q(x)$ given by

$$
q_{1}(x)=\frac{1}{4 \beta^{2}}-\frac{1}{\beta} f^{\prime}\left(u_{1}(x)\right)
$$

are sketched in Fig. 2 and Fig. 3 respectively from the asymptotic approximation given by (2.4), (2.5). (Note that our figures are intended to be roughly accurate illustrations but not precise graphs.) Clearly, we can find numbers $N>0$ and $x_{0} \in[0,1]$ such that

$$
q_{1}(x) \geqq Q(x) \equiv \begin{cases}0 & \text { for } 0 \leqq x \leqq x_{0} \\ -N & \text { for } x_{0}<x \leqq 1\end{cases}
$$




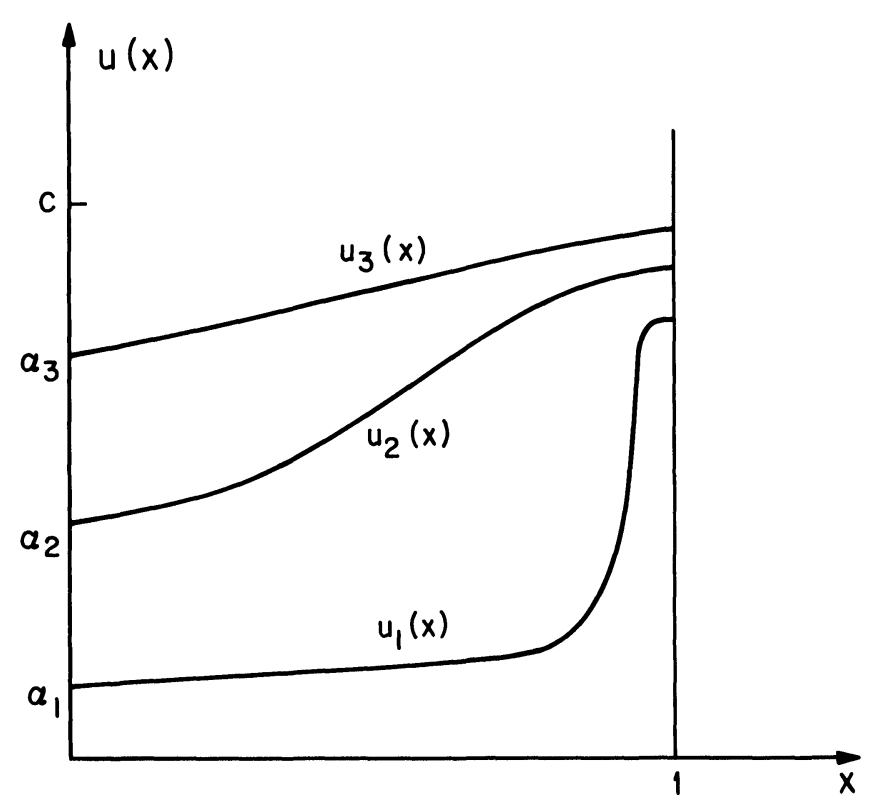

FIG. 2

From the variational characterization of the first eigenvalue $\sigma_{1}$ of the problem (4.12)-(4.15) we can write that

$$
\begin{aligned}
& \sigma_{1}=\min _{\phi(x) \in \mathcal{M}}\left[\frac{\int_{0}^{1} \phi^{\prime 2} d x+\int_{0}^{1} q_{1} \phi^{2} d x+\left(a-\frac{1}{2 \beta}\right) \phi^{2}(0)+\frac{1}{2 \beta} \phi^{2}(1)}{\int_{0}^{1} \phi^{2} d x}\right] \\
& \underset{\quad(4.19)}{\geqq \min _{\phi(x) \in \mathcal{M}}}\left[\frac{\int_{0}^{x_{0}} \phi^{\prime 2} d x+\int_{x_{0}}^{1} \phi^{\prime 2} d x-N \int_{x_{0}}^{1} \phi^{2} d x+\left(a-\frac{1}{2 \beta}\right) \phi^{2}(0)+\frac{1}{2 \beta} \phi^{2}(1)}{\int_{0}^{1} \phi^{2} d x}\right] \\
& \geqq \min _{\phi(x) \in \mathcal{M}}\left[\frac{\int_{0}^{x_{0}} \phi^{\prime 2} d x+\mu_{1} \int_{x_{0}}^{1} \phi^{2} d x-N \int_{x_{0}}^{1} \phi^{2} d x+\left(a-\frac{1}{2 \beta}\right) \phi^{2}(0)}{\int_{0}^{1} \phi^{2} d x}\right],
\end{aligned}
$$

where the class $\mathscr{M}$ of admissible functions consists of all functions $\phi(x)$ continuous on $0 \leqq x \leqq 1$ and continuous differentiable on $0<x<1$, and where $\mu_{1}$ is the first eigenvalue of the problem

$$
\begin{aligned}
& \psi^{\prime \prime}+\mu \psi=0, \\
& x_{0}<x<1 \text {, } \\
& \psi^{\prime}\left(x_{0}\right)=0,
\end{aligned}
$$$$
\psi^{\prime}(1)+(1 / 2 \beta) \psi(1)=0 \text {. }
$$ 


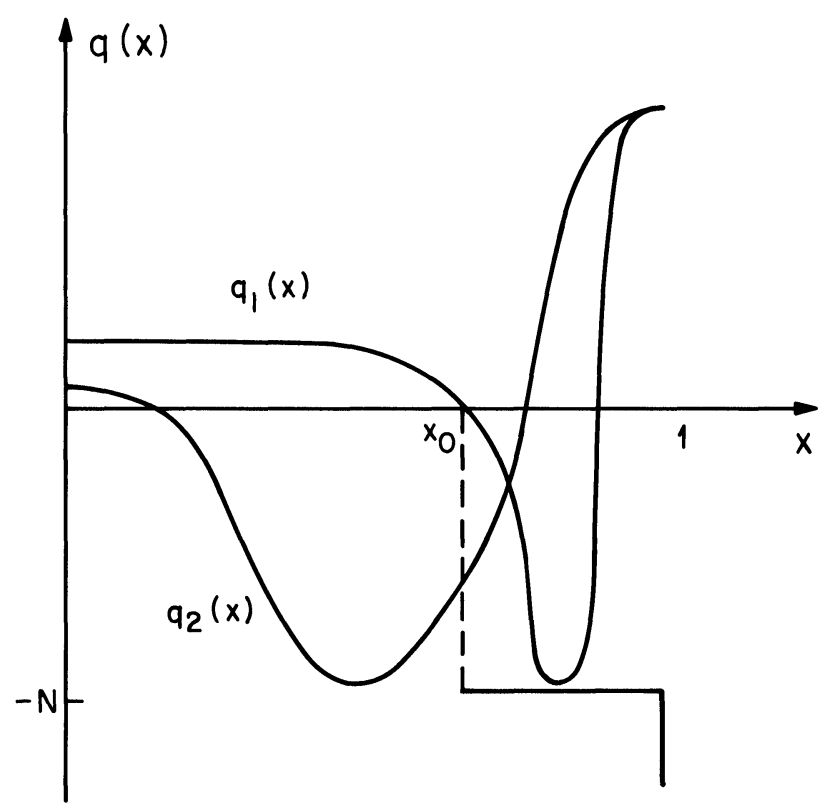

FIG. 3

The last inequality in (4.19) follows from using the variational characterization of $\mu_{1}$. The last quantity in (4.19) is positive if $\mu_{1}>N$, and thus we conclude that $\sigma_{1}>0$ if $\mu_{1}>N$.

The eigenvalues $\mu_{n}$ of (4.20)-(4.22) are the roots of

$$
\tan \left(1-x_{0}\right) \mu^{1 / 2}=\frac{1}{2 \beta \mu^{1 / 2}} .
$$

The mechanism governing stability is now clear. The eigenvalue $\mu_{1}$ increases as $1-x_{0}$ decreases. Hence, the inequality $\mu_{1}>N$ expresses the relationship between the magnitude of the negative values of $q_{1}(x)$ and the length of the interval on which they occur. (Clearly, the best choice for $-N$ is the minimum of $q(x)$; that is,

$$
N=\frac{-1}{4 \beta^{2}}+\frac{1}{\beta} f^{\prime}(\xi)
$$

where $f^{\prime \prime}(\xi)=0$.)

Let $x_{0}$ denote the first positive zero of $q_{1}(x)$, and let $u_{0}=u\left(x_{0}\right)$. Thus,

$$
q_{1}\left(x_{0}\right)=\frac{1}{4 \beta^{2}}-\frac{1}{\beta} f^{\prime}\left(u_{0}\right)=0 .
$$

Since $f^{\prime}(\xi)=O(1 / \beta)$, we can let $N=l / \beta^{2}$. (Actually from experimental values of the parameters [10], [12] it is often the case that $1<l<10$, or independent of the practical chemical reactor theory we can force this to be the case by adjusting values of $\beta, b, c$ and $k$.) Replacing $\tan x$ by its power series and solving (4.23) to first 
order in $\beta$, we find that

$$
\mu_{1}=\frac{1}{2\left(1-x_{0}\right) \beta} \text { if } x_{0}>1-12 \beta^{2} .
$$

Now, $\mu_{1}>N$ implies that $x_{0}>1-\beta /(2 l)$, and for $\beta$ sufficiently small we have $1-12 \beta^{2}>1-\beta /(2 l)$. Therefore,

$$
\mu_{1}>N \text { if } x_{0}>1-12 \beta^{2} \text {. }
$$

Now, as we showed in $\S 2$, to first order in $\beta$, the asymptotic expansion of $u_{1}(x)$ on $0 \leqq x \leqq 1$ is given by

$$
\begin{aligned}
v^{\prime} & =f(v), \\
v(0) & =\alpha_{1},
\end{aligned}
$$

which immediately implies

$$
x=\int_{\alpha_{1}}^{v\left(x_{0}\right)} \frac{d v}{f(v)} .
$$

For various values of $\alpha_{1}$ the solutions of (4.28), (4.29) or equivalently, the curves given by (4.30), are sketched in Fig. 4. We can now conclude that if $\alpha_{1}$ is such that $0<\alpha_{1}<\alpha_{0}$ (where the definition of $\alpha_{0}$ is clear from Fig. 4) then the solution $u_{1}(x)$ is stable. This follows from the facts that on any curve emanating from an $\alpha_{1}$ in $0<\alpha_{1}<\alpha_{0}$ the inequality $x_{0}>1-12 \beta^{2}$ is satisfied and simultaneously $u(x)$ $<u_{0}$, the first value where $q_{1}(x)$ becomes negative.

Therefore, by employing the asymptotic description (2.4), (2.5), we have an easily applicable sufficient condition for stability. For fixed $\beta$ and a given function (1.4) the numbers $l$ and $u_{0}$ are easily calculated. The integral curves of (2.4), (2.5) are then plotted, and thus a figure like Fig. 4 is generated from which the values of

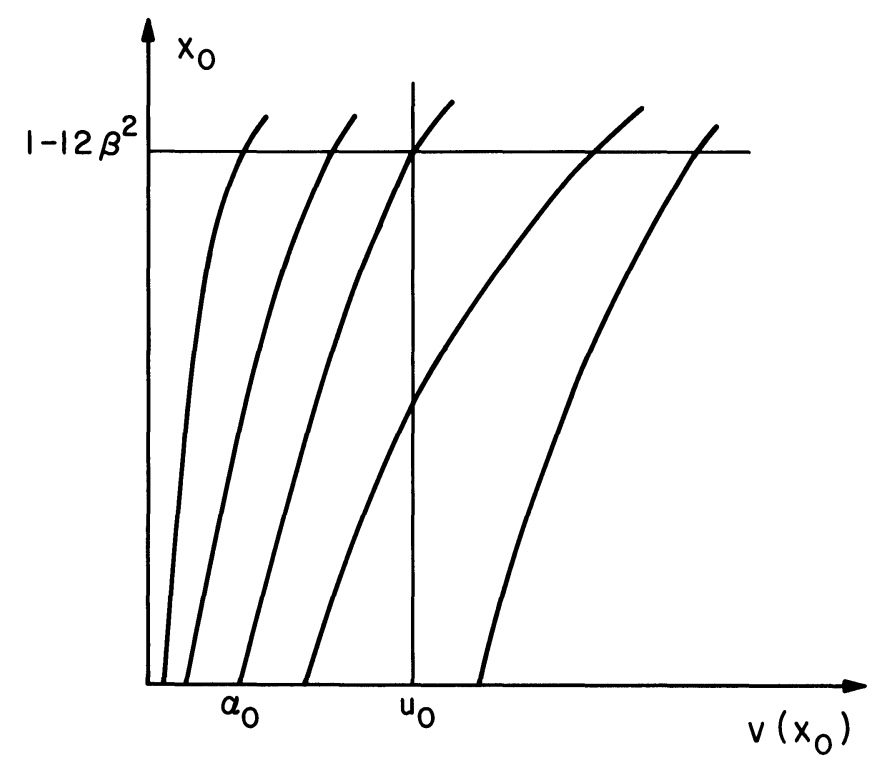

FIG. 4 
$\alpha_{1}$ sufficient for stability are immediately obvious. Furthermore, if an $\alpha=\tilde{\alpha}$ yields a stable solution, then clearly all values of $\alpha$ in $0<\alpha \leqq \tilde{\alpha}$ also yield a stable solution. This last fact is useful in practice because the flow rate (and hence $\beta$ and $a$ ) and $k$ are easily controlled and changed, and as we saw in $\S 2$ these changes cause changes in the intersection point $\alpha_{i}$.

One final fact we wish to point out is that no matter how small $\beta$ and $u_{0}$ we can always adjust $k$ so that there always exist curves (as in Fig. 4) such that on them we simultaneously satisfy the inequalities $x_{0}>1-12 \beta^{2}$ and $v\left(x_{0}\right)<u_{0}$. This follows because

$$
\int_{0}^{u_{0}} \frac{d v}{f(v)}>\frac{e^{k /(1+c)}}{b} \int_{0}^{u_{0}} \frac{d v}{c-v}=\frac{e^{k /(1+c)}}{b} \ln \left(\frac{c}{c-u_{0}}\right)
$$

and for any $u_{0}>0$ we can find a $k$ sufficiently large that this last quantity exceeds unity.

Numerical results for very small values of $\beta$ are given in Example 3 and Fig. 10 of Amundson and Luss [12]. For $0<\beta<0.03$ the stability of the upper and lower of the three solutions computed can be demonstrated by following our procedures. By direct computation we can show that for the lower solution $q(x)$ is positive and for the upper solution $q(x)$ is negative on a sufficiently short interval so that $\mu_{1}>N$.

We have been able to show that the upper and lower solutions are stable only for special ranges of the parameters. However, we believe that the same is true in all cases where three solutions exist, and thus, we conjecture that our iteration schemes of $\S 3$ always converge to stable solutions.

Acknowledgment. The author would like to express his appreciation to Professor Erik B. Hansen at the Technical University of Denmark for the many discussions and critical readings of this manuscript which greatly facilitated and improved the paper.

\section{REFERENCES}

[1] R. ARIS, On stability criteria of chemical reaction engineering, Chem. Engrg. Sci., 24 (1968), pp. 149-169.

[2] D. Luss, Sufficient conditions for uniqueness of the steady state solutions in distributed parameter systems, Ibid., 23 (1968), pp. 1249-1255.

[3] J. D. Cole, Perturbation Methods in Applied Mathematics, Blaisdell, Waltham, Mass., 1968.

[4] M. H. Protter and H. F. Weinberger, Maximum Principles in Differential Equations, PrenticeHall, Englewood Cliffs, N.J., 1967.

[5] D. S. COHEN AND T. W. LAETSCH, Nonlinear boundary value problems suggested by chemical reactor theory, J. Differential Equations, to appear.

[6] R. B. Simpson AND D. S. CoHEN, Positive solutions of nonlinear elliptic eigenvalue problems, J. Math. Mech., to appear.

[7] H. B. Keller ANd D. S. Cohen, Some positone problems suggested by nonlinear heat generation, Ibid., to appear.

[8] H. B. Keller, Elliptic boundary value problems suggested by nonlinear diffusion processes, Arch. Rational Mech. Anal., to appear.

[9] ㄴ... Positive solutions of some nonlinear eigenvalue problems, J. Math. Mech., to appear.

[10] L. R. Raymond And N. R. Amundson, Some observations on tubular reactor stability, Canad. J. Chem. Engrg., 42 (1964), pp. 173-177.

[11] N. R. Amundson, Some further observations on tubular reactor stability, Ibid., 43 (1965), pp. $49-55$.

[12] N. R. AMUNDSON AND D. Luss, Qualitative and quantitative observations on the tubular reactor, Ibid., 46 (1968), pp. $424-433$. 